\title{
Inbound Recruiting y su influencia en la resiliencia en postulantes de la empresa W2 Asesores y Consultores
}

\author{
Inbound Recruiting and its influence on resilience in \\ applicants of the company W2 Asesores y Consultores
}

\begin{abstract}
RESUMEN
El objetivo de este artículo es determinar la influencia del Inbound Recruiting sobre la Resiliencia en postulantes a la empresa W2 Asesores y Consultores. El Inbound Recruiting es una metodología novedosa de reclutamiento y selección de personal que procede del Inbound Marketing, que está enfocado en el cliente, en este caso, los candidatos; quienes en su mayoría se encuentran en condición de desempleados, por lo tanto, con efectos negativos en el ámbito personal y social. En este sentido, es necesario comprender la influencia directa que existe entre estos dos conceptos: Inbound Recruiting y Resiliencia; de este modo puedan ser replicados por diversas organizaciones. Este estudio estuvo constituido por 314 postulantes a procesos operativos, a quienes se aplicó el cuestionario adaptado de Inbound Recruiting, y Resiliencia. El alcance de la investigación es descriptivo-correlacional. En los resultados se evidencia que existe una correlación moderada de tipo positiva ( $\mathrm{Rho}=.449^{* *} ; \mathrm{p}=.000$ ), dando a conocer que a mayores puntajes en Inbound Recruiting, mayores serán los puntajes en Resiliencia.
\end{abstract}

Palabras Clave: Reclutamiento; Inbound Recruiting; Resiliencia; Desempleo.

\begin{abstract}
The objective of this article is to determine the influence of Inbound Recruiting on Resilience in applicants to the company W2 Asesores y Consultores. Inbound Recruiting is a new methodology for recruiting and selecting personnel that comes from Inbound Marketing, which is focused on the client, in this case, the candidates; who are mostly unemployed, therefore with negative effects in the personal and social sphere. In this sense, it is necessary to understand the direct influence that exists between these two concepts, Inbound Recruiting and Resilience, so that they can be replicated by various organizations. This study consisted of 314 applicants to operational processes, to whom the questionnaire adapted from Inbound Recruiting, and Resilience was applied. The scope of the research is descriptive-correlational. The results show that there is a
\end{abstract}

Presentado: 10/03/2021 - Aceptado: 31/08/2021 - Publicado: 23/12/2021

(C) Los autores. Este artículo es publicado por la revista Gestión en el Tercer Milenio de la Facultad de Ciencias Administrativas Universidad Nacional Mayor de San Marcos. Este es un artículo de acceso abierto, distribuido bajo los términos de la licencia Creative Commons Atribución 4.0 Internacional (CC BY 4.0) [https://creativecommons.org/licenses/by/4.0/deed.es] que permite el uso, distribución y reproducción en cualquier medio, siempre que la obra original sea debidamente citada de su fuente original. 
moderate positive correlation ( $\mathrm{Rho}=.449^{* *} ; \mathrm{p}=.000$ ), showing that the higher the Inbound Recruiting scores, the higher the Resilience scores.

Keywords: Recruitment; Inbound Recruiting; Resilience; unemployment.

\section{INTRODUCCIÓN}

Durante los últimos años la manera en el que las personas viven y trabajan fue cambiando drásticamente, siendo la tecnología el principal protagonista. Los colaboradores estiman otros aspectos y no solo sueldo, desean sentirse motivados y ser parte de un equipo en un proyecto determinado. El ámbito laboral, también se encuentra en constante cambio. Pues no solo las organizaciones buscan nuevos talentos y habilidades, sino que la estrategia de las organizaciones para encontrar a sus talentos ideales se ha convertido al uso de nuevas tecnologías. Con la venida de nuevas tecnologías el aspecto de la vida personal y organizacional ha cambiado. Es por ello que ha variado la forma en que los candidatos manejan sus carreras y buscan trabajo.

Según Reyes (2019), en el reporte realizado el 28 de agosto de 2019 en el diario GESTIÓN en el último informe emitido por el Instituto Nacional de Estadística e Informática (INEI) que recoge información del mercado laboral tanto formal como informal, nos muestra que 2.4 semanas fueron el promedio de tiempo de desempleo. Quienes tuvieron facilidad de encontrar empleo con mayor rapidez fueron aquellos entre 14 a 24 años, 2.2 semanas promedio en búsqueda, a los de 25 a 44 años les tomó 2.5 semanas promedio. Y a los de 45 a más les tardó 2.6 semanas promedio para lograr un nuevo empleo. Teniendo en cuenta estos indicadores de desempleo podemos afirmar que frente al desempleo el bienestar psicológico de una persona es vulnerado, pues afronta inestabilidad que afecta la percepción que tiene el individuo sobre su autoestima, las relaciones interpersonales, su desarrollo personal, misión de vida y autonomía.

Según Estramiana et al. (2012) en el estudio de investigación sobre Desempleo y Bienestar Psicológico en Brasil y España. El fin de este estudio fue entender la relación entre el desempleo y el bienestar psicológico contrastando dos muestras una de trabajadores empleados y la otra de trabajadores desempleados. Se obtuvo como resultado que el desempleo está relacionado a un deterioro del bienestar psicológico y estas diferencias no tienen relación con el género, la edad y el tiempo de desempleo.

Lévano (2018) en su tesis titulada Resiliencia laboral en los trabajadores de una institución educativa del distrito de San Miguel por la Universidad Inca Garcilaso de la Vega, Lima, Perú. El fin de este estudio fue determinar los niveles de Resiliencia laboral y las dimensiones en colaboradores dentro de una institución educativa. Los resultados nos demuestran la intensidad del nivel medio alto en resiliencia laboral en los colaboradores. Pero, en las dimensiones el nivel promedio es bajo, no existiendo una diferencia significativa en todos los casos.

Finalmente Murillo (2015) en su tesis que lleva por título Sistemas de reclutamiento y selección del personal para la cadena de supermercados MERCAMAXX del Canton Los Bancos, provincia de Pichincha - Ecuador, tuvo el fin de optimizar el desempeño laboral en los supermercados por medio de la elaboración de una secuencia de etapas de reclutamiento y selección de personal que permita mejorar el desempeño laboral; con los resultados se concluyeron que un desarrollo inadecuado, sin etapas ni procesos definidos de reclutamiento y selección de personal, tiene como consecuencia errores en la contratación de personal, por ende un bajo desempeño laboral lo cual genera una baja eficiencia en la producción de una empresa.

En W2 Asesores y Consultores, desde el tercer trimestre del 2019, se implementó la metodología Inbound Recruiting en el que las estrategias del inbound Marketing se tornan relevantes al momento de reclutar, siento no solo eficiente sino eficaz, el inbound Marketing fue creado por el cofundador y CEO de HubSpot, Halligan y Shah (2005) y adaptado al reclutamiento de personal por la empresa Talent Clue, con el objetivo de captar a los candidatos con talento y potencial, y los mismos se vean interesados en ser parte de la organización ya sea que estén buscando empleo o que no lo requieran. 
El futuro de las estrategias de atracción de talento humano hoy en día es dirigirse a los candidatos como si de futuros clientes se tratara.

Tomando en cuenta lo antes mencionado, con el presente estudio se busca identificar la influencia del Inbound Recruiting sobre la Resiliencia en postulantes en la empresa W2 Asesores y Consultores; considerando que actualmente el talento humano es quien mueve el mercado laboral, el Inbound Recruiting se torna una estrategia de atracción de talento Humano y contratación a largo plazo; y ésta se enfoca en desarrollar la marca del empleador lo cual se traduce en el incremento de solicitantes de empleo de calidad.

\section{Reclutamiento de personal}

Según Alles (2006), El reclutamiento es una serie de procedimientos que tienen como fin la atracción e identificación de candidatos calificados con la finalidad de ocupar el cargo solicitado por la empresa.

Llanos (2005) refiere que el reclutamiento es uno de los procesos de mayor importancia dentro de las organizaciones, debido a que "de ello depende que la organización cuente con personal calificado, actualizado y capacitado" (p. 79), y a la vez "le otorga un nivel de competitividad y cumplimiento cabal de sus objetivos globales" (p. 79). En las áreas de Gestión Humana de las organizaciones, la atracción de personal (reclutamiento) se ha tornado un proceso muy levante para atraer a los mejores talentos. El reclutamiento se puede entender como un proceso direccionado a buscar candidatos potenciales para ocupar las vacantes actuales que se tiene en una organización.

\section{Inbound Marketing}

Halligan y Shah (2010) empezaron HubSpot, una compañía de software acerca de Inbound Marketing con la misión de apoyar a empresas a hacer uso y apoyarse de la revolución digital para obtener beneficios por el mismo. El Inbound Marketing hace uso de herramientas digitales para cautivar a clientes, por lo que es considerado como una estrategia de marketing, de incitación. Las organizaciones dejan vender de forma invasiva y empiezan a cautivar a su cliente ideal. Es de esta forma que la comunicación digital a través del Inbound Marketing busca atraer clientes de una manera más creativa y sutil. Por lo que, en reconocimiento a profesionales de marketing que experimentaron con sus herramientas y fueron minuciosos al observar lo que realizaban, hoy se pudo dividir según ellos en dos tendencias de mercadeo diferenciadas: el Outbound Marketing y el Inbound Marketing.

En una encuesta realizada por HubSpot en el año 2013 se evidencia que el 58\% de empresas en el mundo ya usan este método, por lo que estas empresas incrementaron su presupuesto en un 50\%; otra información importante que se pudo obtener es que 1 de cada 3 potenciales clientes, tiene la característica de cooperar debido a la estrategia de Inbound Marketing. Según Valdez (2014) el Inbound Marketing tiene como objetivo incrementar las visitas en una página web, un perfil o blog en redes sociales, por medio de técnicas de marketing digital, llevándolos finalmente a ser seguidores de los mismos, quiere decir, registro de datos de las personas interesadas en el contenido digital de la organización.

\section{Inbound Recruiting}

Una de las empresas pioneras en efectuar la metodología llamada Inbound Recruiting basada en el Inbound Marketing, es Talent Clue. Esta metodología tiene como finalidad hacer del Marketing y los procesos atracción de personal sean una experiencia diferenciada para los potenciales colaboradores de la organización. Consiste en atraer a los futuros colaboradores por medio del marketing y esforzarse en captar su atención para finalmente cautivarlos y hacerlos desear formar parte de la organización. En concreto se busca humanizar los procesos de atracción de personal y modificar el estilo de relación que se tiene con los colaboradores y futuros colaboradores.

Según Sala e Izquierdo (2013), El Inbound Recruiting permite visibilizar la imagen de la organización como el lugar ideal para laborar y esto poder comunicarlo por los canales más adecuados para atraer a candidatos talento. Siendo así, una organización tendrá la facilidad de conseguir candidatos calificados, de forma continua e independientemente si en la organización existen requerimientos por cubrir en 
el momento. El reto de esta estrategia es involucrar a las personas y posibles colaboradores, para luego convertirlos en postulantes a las ofertas laborales de la organización. Empezar y crear relaciones con los postulantes, para darles a conocer sobre la organización y la propuesta de valor en cada momento de contacto. Es de esta forma, que se crea un trato de confianza entre los postulantes y la organización mucho antes de tener un primer contacto. Por lo tanto, en un ámbito promovido por el talento, las organizaciones requieren ser proactivas para atraerlos.

Porque el éxito del reclutamiento no es simplemente publicar ofertas laborales en diversas bolsas de trabajo o portales y de forma pasiva esperar que gran cantidad de postulantes se interesen. El éxito está en formar relaciones entre la organización y el candidato haciendo que se sienta atraído por la misma. Pues las personas con gran talento generalmente son atraídas por organizaciones con una cultura sólida.

Fases del Inbound Recruiting (ver Figura 1):

- Atraer: Se atrae al talento (candidatos) perfilados a la cultura de la organización.

- Convertir: Se convierte de la manera más segura la visita de un talento en candidato.

- Contrata: Se contrata con mayor rapidez y menores recursos.
- Enamora: Se enamora a los candidatos en todo el proceso

\section{Resiliencia}

La concepción de resiliencia aún continúa en evolución, debido a que varios autores proponen definiciones acordes a las circunstancias y evolución del ser humano, es así que Yaria (2005) sustenta que, la forma en cómo el ser humano traza estrategias constructivas que le permitan solucionar conflictos y aprender de ellos para vivir con los mismos, se le llama resiliencia. Wolin y Wolin (1993) mencionan que es la capacidad del ser humano para superar una situación difícil, forjando procesos internos que fortalecen y favorecen las capacidades del ser humano.

Wagnild y Young (1993) la definen como la particularidad de la personalidad que concede vigor y fortaleza emocional que suscita recursos internos moduladores del efecto perjudicial de experiencias traumáticas, admitiendo la tolerancia, reestructuración y finalmente la adaptación emocional, mental, y física, del individuo que lo experimenta, es así como se consuma en un fortalecimiento positivo del yo. Además, acotan que "es una cualidad de personalidad que modera el efecto negativo del estrés y fomenta la adaptación. Ello connota vigor emocional y es usado para describir a sujetos que muestran valentía y adaptabilidad ante los infortunios de la vida" (p. 06).

\section{Figura 1}

Proceso de la Metodología Inbound Recruiting

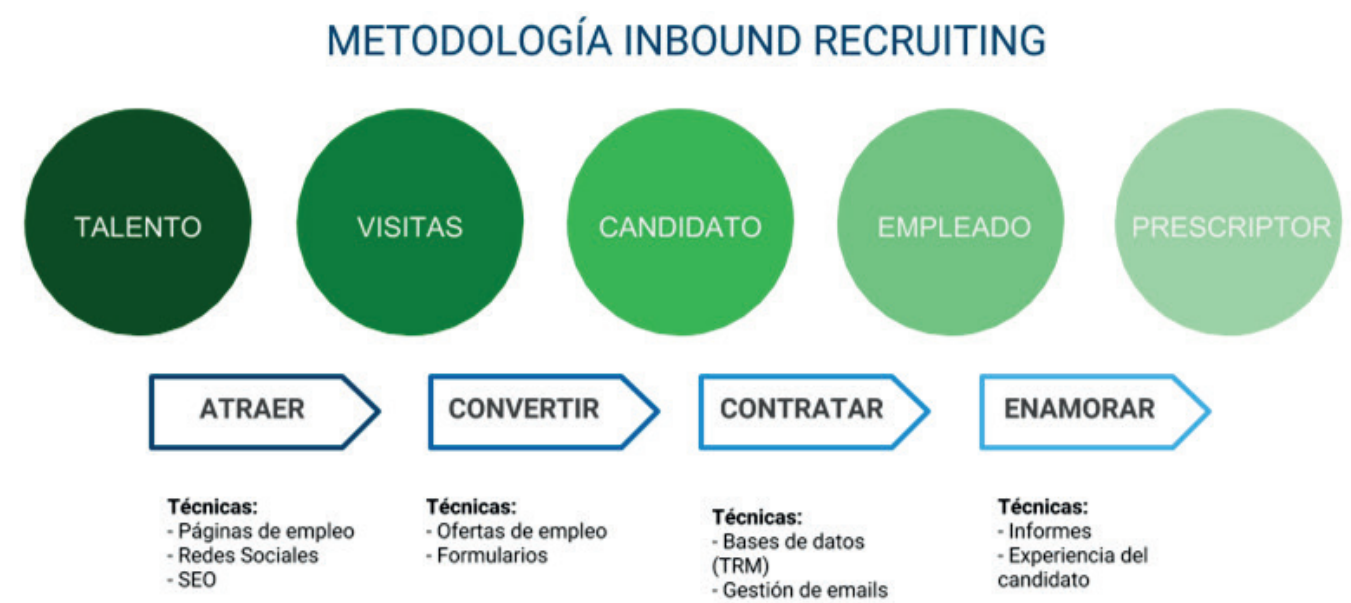

Nota. Esquema del proceso de atracción en Inbound Recruiting y las técnicas que se utilizan en cada fase. Tomado de Comunica web "Inbound Recruiting qué es, definición" https://comunica-web.com/blog/marketing-digital/inbound-recruiting-que-es-definicion/ 
Munist y Suárez (2007) refieren que es la posición humana, que sugiere la capacidad para aguantar y superar situaciones adversas y hostiles, y que incluso es posible que haga uso de esas situaciones para realizar cosas extraordinarias. Dicho de otra manera, se entiende como el proceso de adaptación a situaciones hostiles.

Es por ello que se entiende como resiliencia a la concentración de procesos intrapsíquicos y sociales que, a lo largo del tiempo, le permite al ser humano tener una vida sana y poder desarrollarse de manera favorable a pesar de encontrarse en ámbitos disruptivos. En efecto la resiliencia es la capacidad que se adquiere en las situaciones desfavorables que ocurren a lo largo de la vida, y no es algo con lo que se nace (Villalba, 2014).

\section{MÉTODOS}

Considerando el marco conceptual de Hernández (2014) el presente artículo tiene un enfoque cuantitativo, toda vez que de la aplicación del instrumento de recolección de datos de las diferentes variables se obtendrán resultados numéricos, los mismos que se procesarán estadísticamente, el alcance descriptivo de tipo correlacional que mide la intensidad de esa relación entre las variables, así como el grado de relación que existe entre ellas.
El diseño correlacional tiene como esquema el siguiente:

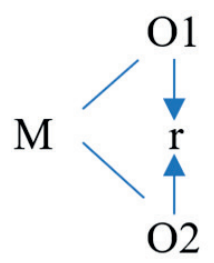

Donde:

$\mathrm{M}=$ Representa la muestra en estudio.

$\mathrm{O}_{1}=$ Inbound Recruiting

$\mathrm{O}_{2}=$ Resiliencia

$r$ = Relación entre las dos variables

Este artículo científico se basó en la investigación que se realizó el año 2020, cuya selección de muestra es probabilística; la población de la presente investigación fue de 1400 postulantes a procesos operativos, haciendo uso del nivel de confianza de 95\% con un margen de error del $5 \%$ para el total de postulantes; teniendo como resultado una muestra de 314 postulantes.

En la siguiente Tabla 1, se observan las características idiosincráticas más representativas de los participantes.

Tabla 1

Variables sociodemográficas $(n=314)$

\begin{tabular}{|c|c|c|c|c|c|}
\hline Variable & $\mathbf{f}$ & $\%$ & $\mathbf{M}$ & Mo & DE \\
\hline \multicolumn{6}{|l|}{ Sexo } \\
\hline Hombre & 312 & 99.4 & \multirow{2}{*}{ - } & \multirow{2}{*}{1} & \multirow{2}{*}{-} \\
\hline Mujer & 2 & 0.60 & & & \\
\hline \multicolumn{6}{|l|}{ Edad } \\
\hline 18 a 25 & 73 & 23.2 & \multirow{4}{*}{19.5} & \multirow{4}{*}{2} & \multirow{4}{*}{.079} \\
\hline 26 a 35 & 127 & 40.4 & & & \\
\hline 36 a 50 & 98 & 31.2 & & & \\
\hline 51 a más & 16 & 5.10 & & & \\
\hline \multicolumn{6}{|l|}{ Grado de instrucción } \\
\hline Secundaria & 308 & 98.1 & \multirow{4}{*}{ - } & \multirow{4}{*}{1} & \multirow{4}{*}{ - } \\
\hline Técnico & 2 & 0.60 & & & \\
\hline Técnico trunco & 3 & 1.00 & & & \\
\hline Universitario trunco & 1 & 0.30 & & & \\
\hline \multicolumn{6}{|l|}{ Experiencia } \\
\hline 0 a 5 años & 234 & 74.5 & \multirow{5}{*}{ - } & \multirow{5}{*}{1} & \multirow{5}{*}{-} \\
\hline 6 a 10 años & 59 & 18.8 & & & \\
\hline 11 a 15 años & 17 & 5.40 & & & \\
\hline 16 a 20 años & 2 & 0.60 & & & \\
\hline 21 a más & 2 & 0.60 & & & \\
\hline
\end{tabular}

Nota. f = Frecuencia, \% = Porcentaje, $\mathrm{M}=$ Media, $\mathrm{Mo}=$ Moda, DE = Desviación Estándar, -: Ausencia de valor. Elaboración propia. 
Se hizo uso de la técnica de la encuesta que según De la Cruz, I., et. al. (2006) refieren que nos permite indagar, explorar y recolectar datos, ello por medio de preguntas estructuradas directa o indirectamente a los individuos de la muestra. Considerando como variable independiente al Inbound Recruiting y como variable dependiente a la Resiliencia, se realizó la adaptación de las encuestas por cada una de las variables, ambos instrumentos pasaron un proceso de validez y confiabilidad; validez a través del método de análisis de jueces mediante el Coeficiente de Validez $\mathrm{V}$ de Aiken, siendo el resultado $100 \%$ válidos. Y confiabilidad a través del método de consistencia interna coeficiente alfa de Cronbach, obteniendo el resultado de .878 para el cuestionario de Inbound Recruiting y .823 para el cuestionario de Resiliencia. Estos datos permitieron conocer la excelente fiabilidad de los instrumentos aplicados.

\section{RESULTADOS}

Dado que, la muestra es mayor a los 50 participantes (Verma, 2019). Por ende, para los siguientes resultados se hizo uso de las pruebas no paramétricas mediante el coeficiente de correlación de Spearman. Teniendo en cuenta el análisis de la prueba de normalidad de nuestra muestra, se procede a realizar la prueba de $\mathrm{Hi}$ pótesis General, con el Coeficiente de Correlación Rho Spearman, teniendo los siguientes resultados como se presenta en la Tabla 2.

Tabla 2

Correlación entre la Inbound Recruiting y Resiliencia ( $n=$ 314)

\begin{tabular}{lll}
\hline Variable & Coe. & Resiliencia \\
\hline \multirow{2}{*}{ Inbound Recruiting } & Rho & $.449^{* *}$ \\
& $p$ & .000 \\
\hline
\end{tabular}

Nota. ${ }^{* *}$. La correlación es significativa en el nivel 0,01 (bilateral), Rho $=$ Coeficiente de Spearman, $\mathrm{p}=$ Significancia estadística. Ela boración propia.

En la Tabla 2, se observa el resultado de la hipótesis general, igual a Rho $=.449$ que evidencia la relación positiva de nivel moderado entre el Inbound Recruiting y la Resiliencia, siendo el nivel de significancia bilateral igual a $\mathrm{p}=.000$; por lo tanto, se rechaza la hipótesis nula y se acepta la hipótesis alterna, que señala: el Inbound Recruiting se relaciona con la resiliencia en postulantes en la empresa W2 Asesores y Consultores en el año 2020.

Como si fuera poco, se adicionó la correlación mediante el modelo de ecuaciones estructurales teniendo los siguientes resultados presentados en la Tabla 3.

Tabla 3

Correlación entre variables mediante SEM $(n=314)$

\begin{tabular}{lccc}
\hline Correlaciones & $\boldsymbol{\lambda}$ & $\mathbf{D E}$ & $\mathbf{p}$ \\
\hline Inbound recruiting $\leftrightarrow$ Resiliencia & .540 & .012 & $* * *$ \\
\hline
\end{tabular}

Nota. $\lambda$ : Coeficiente de Lambda, DE: Desviación Estándar, n: Muestra, ${ }^{* * *}$ : Estadísticamente significativo, $\mathrm{n}=$ Muestra. Elaboración Propia.

En la Tabla 3 se evidencia adecuados valores entre las variables latentes de Inbound Recruiting y Resiliencia, observándose una relación significativa de tipo positiva $(\lambda=.540)$, es decir, el efecto que causa una variable sobre la otra da como conclusión una relación moderada entre las variables en estudio.

\section{DISCUSIÓN}

La finalidad del presente artículo es demostrar la influencia del Inbound Recruiting sobre la Resiliencia de postulantes en la empresa W2 Asesores y Consultores; y dadas las evidencias presentadas en los resultados, podemos inferir que se alcanzó la meta propuesta al conocer que efectivamente el Inbound Recruiting influye a nivel moderado de manera directa y positiva en la Resiliencia.

Al aplicar el Inbound Recruiting, se mejoran los resultados organizacionales, así como también los aspectos personales de los candidatos, al momento de Atraer se busca hacer visible a la empresa frente al público en general, atraer tráfico a la página de empleo y mantener actividad en redes sociales para generar presencia de la organización. Al momento de Convertir, se busca convertir las visitas a las páginas en futuros candidatos para las posiciones actuales o futuras posiciones a requerir; es importante conservar una comunicación fluida, que facilite la aplicación a los puestos. Esta comunicación clara es la clave de todo el proceso pues, por medio de ella se puede informar al postulante, así como evaluarlo y brindarles un feedback respectivo pues no solo debe estar atraído por la empresa, sino que debe estar seguro a 
que empresa se está postulando. Tomando en cuenta los estudios de Flores (2008) sobre resiliencia y Proyecto de vida en estudiantes de 3er año de secundaria, nos da referencias que la resiliencia tiene relación e impacto significativo en la definición de proyecto de vida. Además, Cuadra y Florenzano (2003) indican que: "el bienestar subjetivo se refiere a lo que las personas piensan y sienten acerca de sus vidas y a las conclusiones cognoscitivas que ellos alcanzan cuanto evalúan su existencia" (p. 83). Teniendo en cuenta ello podemos inferir que el Inbound Recruiting también influye en la definición de proyecto de vida de una persona, por lo tanto, en la mejora de su bienestar subjetivo.

Al momento de Contratar se busca cerrar los procesos de atracción de personal y convertir a los talentos en parte de la empresa, para ello se debe tener una gestión rápida y eficaz por medio de una comunicación personalizada. Finalmente, al momento de Enamorar, se busca que durante todo el proceso de selección se fomente diversas actividades que transmitan a los candidatos el alma de la organización. Si el proceso se dio de manera satisfactoria los colaboradores serán quienes comparan su experiencia con otras personas; de esta manera, no solo se consigue un buen feedback por parte del colaborador, sino que además se estará promocionando a la organización de forma indirecta, lo cual se corrobora con el estudio de Espilco (2018) quien afirma sobre eficiencia de la Metodología del reclutamiento 2.0 comparado con el reclutamiento tradicional, podemos evidencia que existe mayor eficiencia en el reclutamiento 2.0. considerando las diferencias de temporalidad, eficacia y costo de horas hombre.

Por otro lado, las personas al estar bajo circunstancias de desempleo, son propensos a sufrir efectos disruptivos a nivel individual y social. Para la Psicología según Cuadra y Florenzano (2003) manifiestan que "el bienestar subjetivo se refiere a lo que las personas piensan y sienten acerca de sus vidas y a las conclusiones cognoscitivas que ellos alcanzan cuanto evalúan su existencia" (p. 83).

Según Diaz (2001) refiere que:

La dimensión cognoscitiva se refiere a la vida en su totalidad o con respecto a áreas específicas de la vida como la matrimonial o laboral, en tanto la dimensión afectiva está relacionada con la frecuencia e intensidad de las emociones positivas y negativas (p. 572).

Es evidente que la pérdida de empleo provoca modificaciones en la forma en la que una persona concibe su vida, el grado de su felicidad o bienestar subjetivo. En estudios de Di Tella et al. (2001) podemos evidenciar que el nivel obtenido de felicidad de individuos que están desempleados es sumamente menor que el de individuos que mantienen un trabajo, teniendo en cuenta particularidades similares. Según los estudios de Giraldo (2013) sobre resiliencia y bienestar psicológico en personas desempleadas, resulta que existe una relación moderada y alta entre estas dos variables; siendo una de ellas el efecto contrario del desempleo, nos referimos al Bienestar Psicológico, pues este viene a ser vulnerado en el desempleo; sin embargo, a mayores niveles de Resiliencia, frente a situaciones vulnerables como es el desempleo, se incrementa el Bienestar Psicológico. Teniendo en cuenta ello, con nuestro estudio podemos evidenciar que la influencia que causa el uso de la Metodología del Inbound Recruitng sobre la Resiliencia, también influye de manera directa y positiva en el Bienestar Psicológico de las personas desempleadas, en este caso específico de los postulantes.

\section{CONCLUSIONES}

Del estudio realizado se obtuvo como principal conclusión que el Inbound Recruiting Influye a nivel moderado en la Resiliencia de postulantes en la empresa W2 Asesores y Consultores, pues existe una correlación moderada de tipo positiva igual a Rho = .449; siendo el nivel de significancia bilateral igual a $\mathrm{p}=.000$. lo cual nos indica que a medida se mejora la aplicación de la metodología del Inbound Recruiting mejorará el nivel de resiliencia en postulantes.

\section{RECOMENDACIONES}

En la actualidad, son muy pocas las empresas peruanas que hacen uso de la Metodología del Inbound Recruiting, esta metodología se encuentra en una etapa de inicio. Si bien es cierto que esta Metodología se aplicó en W2 Asesores 
y Consultores desde fines de año del 2019 y el estudio se realizó en el 2020, se sugiere realizar mayores estudios e investigaciones debido a que uno de los beneficios de esta Metodología es un trabajo a largo plazo que sólo con el tiempo podríamos medir la correlación con otras variables adicionales como: fidelización de personal, rotación de personal, tiempo de adaptación al trabajo, desempeño laboral, así como clima organizacional.

Para tener mayores evidencias de los beneficios del uso de la Metodología del Inbound Recruiting, se sugiere desarrollar mayores investigaciones dirigidas a conocer los efectos del desarrollo de Metodología del Inbound Recruiting, para lo cual se plantea una alternativa de investigación haciendo uso del diseño experimental con dos grupos: un grupo experimental y un grupo control; al grupo experimental se aplique la Metodología de Inbound Recruiting y al grupo control se aplique la metodología tradicional de reclutamiento; en base a los resultados evidenciar si existe una diferencia significativa al aplicar la metodología del Inbound Recruiting con la metodología tradicional.

\section{REFERENCIAS BIBLIOGRÁFICAS}

Alles, M. (2006). Selección por competencias. Recuperado de: https://ebookcentral.proquest.com/lib/upcsp/reader.action?docI$\mathrm{D}=4849692$ \&query=reclutamiento $+2.0+\#$

Cuadra, H. y Florenzano, R. (2003). El bienestar subjetivo: hacia una psicología positiva. Revista de Psicología de la Universidad de Chile XII (1), 83-96

Comunica web. (28 de diciembre de 2016) Inbound Recruiting qué es, definición. https://comunica-web.com/blog/marketing-digital/inbound-recruiting-que-es-definicion/

De la Cruz, I., Morales, J. y Carrasco, G. (2006) Construcción de un instrumento de evaluación de capacidades en la empresa: Una propuesta metodológica. En las memorias del X Congreso Anual de la Academia de Ciencias Administrativas, A.C. (ACACIA), San Luis Potosí, 17-19 May 2006.

Diaz, G (2001). El bienestar subjetivo: actualidad y perspectivas. Revista Cubana de Medicina General Integral 17(6), 572 - 579.

DITella, R; MacCulloch, R. y Oswald, A. (2001). Preferences over Inflation and Unemployment: Evidence from Surveys of Happiness. American Economic Review 91(1), 335-341
Espilco, I. (2018). Eficiencia de la metodología de reclutamiento 2.0 comparado con un reclutamiento tradicional en una universidad de lima metropolitana - 2016. Universidad De San Martin de Porres. Lima. Recuperado de: http:// www.repositorioacademico.usmp.edu.pe/bitstream/usmp/4261/1/espilco_zic.pdf

Estramiana, J., Gondim, S., Luque, A.; Luna, A. y Dessen, M. (2012), Desempleo y Bienestar Psicológico en Brasil y España: Un Estudio Comparativo. Revista Psicologia: Organizações e Trabalho, 12(1) p. 5-16. Recuperado de: http://pepsic.bvsalud.org/pdf/rpot/v12n1/v12n1a02.pdf

Flores, M. (2008). Resiliencia y proyecto de vida en estudiantes del tercer año de secundaria de la UGEL 03. Tesis de Maestría. Universidad Nacional Mayor de San Marcos. Lima. Perú. Recuperado de http://cybertesis.unmsm.edu. pe/bitstream/handle/cybertesis/621/Flores_ cm.pdf; jsessionid=934F7BDECE952BFA2E8B0A04ECEDA389?sequence $=1$

Giraldo Isaza, M. (2013). Resiliencia y Bienestar Psicológico según la duración del desempleo en una muestra de profesionales colombianos. Revista Interamericana de Psicología, 32(2), pp. 22-37

Halligan y Shah. (2010). Inbound Marketing: Get Found Using Google, Social Media and Blogs. Estados Unidos: John Wiley and Sons, Inc

Hernández, R. (2014). Metodología de la Investigación. Interamericana Editores S.A. de C.V. https://www.uca.ac.cr/wp-content/ uploads/2017/10/Investigacion.pdf

Lévano, M. (2018) Resiliencia laboral en los trabajadores de una institución educativa del distrito de San Miguel. Tesis de la Universidad Inca Garcilaso de la Vega. Lima. Perú, Recuperado de: http://repositorio.uigv.edu.pe/bitstream/handle/20.500.11818/3401/TRAB.SUF. PROF_Milagros\%20Mercedes\%20L\%C3\%A9 vano $\% 20$ Acu $\%$ C $3 \%$ B1a.pdf?sequence $=2 \&$ isAllowed=y

Llanos, J. (2005). Integración de Recursos Humamos, Editorial Trillas.

Murillo, P. T. (2015). Sistema de reclutamiento y selección del personal para la cadena de supermercadors Marcamaxx del Canton Los Bancos, Provincia de Pichincha. Ambato: Universidad Regional Autónoma de los Andes. Recuperado de http://dspace.uniandes.edu.ec/ bitstream/123456789/603/1/TUAEXCOMIEA N013-2015.pdf 
Munist, M. y Suárez, E. (2007). Conceptos generales de resiliencia aplicados a adolescentes y jóvenes. En Munist, Suárez Ojeda, Krauskpf y Silber (Comps.) Adolescencia y resiliencia. Buenos Aires: Paidós

Reyes J. (28 agosto, 2019). Desempleo: ¿Cuánto tiempo le toma a los peruanos encontrar un nuevo trabajo?. GESTIÓN. Recuperado de: https:// gestion.pe/economia/management-empleo/ desempleo-cuanto-tiempo-le-toma-a-los-peruanos-encontrar-un-nuevo-trabajo-noticia/

Sala, I. y Izquierdo, A. (2013). Guía complete de Metodología Inbound Recruiting. Recuperado de: https://cdn2.hubspot.net/hubfs/252575/ guia-completa-metodologia-inbound-recruiting.pdf

Verma, J. P. (2019). Non-parametric Tests for Psychological Data. Statistics and Research Methods in Psychology with Excel, 477-521. https://doi. org/10.1007/978-981-13-3429-0_12
Villalba, C. (2014). El concepto de Resiliencia individual y familiar. Aplicaciones en la Intervención social. Revista de Intervención Psicosocial (12) 3, 283-299

Wolin, S. J. y Wolin, S. (1993). The resilient self: how survivors of troubled families rise above adversity. Nueva York: Villard Books

Wagnild, G.M. y Young, H.M. (1993). Development and psychometric evaluation of the Resilience Scale. Journal of Nursing Measurement, 1(2), 165-178.

Yaria, J. (2005). Drogas, escuela, familia y prevención. Argentina: Editorial Bonum 